\title{
Educational Podcasts in Kinesiology: A Scoping Review
}

\author{
Scott W.T. McNamara, Matthew Shaw, Kylie Wilson, and Angela Cox
}

\begin{abstract}
Educational podcasts are developed specifically for learning purposes. Preliminary research suggests that many college courses and practitioners regularly use educational podcasts and that this medium is a beneficial tool to use to supplement the learning process. However, there is limited scholarly work examining the use of educational podcasts within kinesiology fields. Thus, the purpose of this study was to conduct a scoping review of the literature on the use of educational podcasts in the field of kinesiology. The Preferred Reporting Items for Systematic Reviews extension for Scoping Reviews Checklist guided this investigation. Six databases were searched. Fourteen articles met the full inclusion criteria. Of these, 11 were data-driven research articles, and three were practitioner articles. Much of the research identified lacked critical information related to research design, instrument development, and findings. Thus, the authors recommend that more rigorous research in this area be conducted to discern the impact of educational podcasts within the field of kinesiology.
\end{abstract}

Keywords: e-learning, physical education, sport sciences, supplemental learning

Many governing bodies and societies within the field of kinesiology regularly provide professional development (PD), which is perceived as a beneficial mechanism for knowledge sharing and dissemination. However, practitioners and college students often encounter significant barriers to attending PD. For example, York, Gastin, and Dawson (2014) interviewed six sport scientists about their PD attendance, and it was found that although attendance was perceived highly worthwhile in developing their professional network, it came at both a time and financial cost. It is well established that practitioners and college students within the field of kinesiology value different forms of learning, and a body of literature exists, which suggests that informal learning situations are valued more than formal education programs (e.g., Beddoes, Prusak, \& Barney, 2019; Blackett, Evans, \& Piggott, 2017). Online settings and e-learning tools may offer a viable alternative learning opportunity for practitioners and students within the field of kinesiology to engage with relevant content that may have been previously available only to them through professional conferences or face-to-face college courses.

Online learning settings differ from traditional face-to-face learning as it offers the ability to overcome barriers related to accessibility, cost, and a lack of experts in a geographical area (Valian \& Emami, 2013). There is a growing amount of research demonstrating the effectiveness of learning within online settings for bridging knowledge gaps among a variety of professional disciplines (e.g., Healy, Block, \& Kelly, 2020; Valian \& Emami, 2013). For example, Sato and Haegele (2018) investigated physical educators' engagement within an online PD program that focused on adapted physical education (PE) concepts. The online PD was developed using well-established adult learning principles. Through the use of interviews, peer-review feedback assignments, and reflection logs, it was established that the participants had positive and meaningful engagements within the online PD. This study demonstrates that learning within online settings, when well designed,

McNamara, Wilson, and Cox are with the Kinesiology Dept., University of Northern Iowa, Cedar Falls, IA, USA. Shaw is with Høgskulen på Vestlandet, Bergen, Norway. McNamara (scott.mcnamara@uni.edu) is corresponding author. can provide significant learning opportunities that could substitute traditional face-to-face learning. Although the use of learning within online settings has been widespread within the field of kinesiology, it has also been suggested that there is a lack of research examining how to implement specific mechanisms and components associated with learning in these settings (McNamara \& Haegele, 2020; Sato \& Haegele, 2018).

\section{Educational Podcasts}

Podcasts deliver digital content through audio and visual mediums. Podcasts often are dispersed via the Internet through a variety of media devices, such as laptops and smartphones (Hew, 2008). In addition, podcasts have become an increasingly prevalent e-learning tool (Drew, 2017; Healy et al., 2020; Kay, 2012; Kennedy, Rodgers, Romig, Mathews, \& Peeples, 2017; Mcnamara, Dillon, Becker, Healy, \& Trujillo-Jenks, 2020). In this context, one of the most widely reported reasons for podcast usage is its flexibility and personalization (Healy et al., 2020). Podcasts are an educational phenomenon, with social and "media-rich" features that exemplify the uniqueness and broad appeal of learning within online settings.

"Educational podcast" is a commonly used term to describe podcasts that are specifically designed to impact learning (Drew, 2017; Mcnamara, et al., 2020). Educational podcasts are often produced by a teacher as a component of formal online learning materials, or they can be open-access podcasts, which are podcasts that are freely available to the public (Fronek, Boddy, Chenoweth, \& Clark, 2016; McNamara \& Haegele, 2020). This medium enables listeners to be more in control of their learning, as podcasts allow the listener to pause, rewind, and listen to content as often as necessary to properly learn the content (Drew, 2017; Kay, 2012). Educational podcasts, especially open-access podcasts, also have the ability to increase relationships and deepen learners' communities of practice (Lave, Wenger, \& Wenger, 1991), which are often absent in online settings. More precisely, educational podcasts can be an informal method of disseminating profession-specific knowledge and helping to "build stronger relationships between theory, research, and practice in the space between formal learning and entertainment" (Fronek et al., 2016, p. 113). Listening to educational 
podcasts allows listeners to hear the concerns and strategies of likeminded practitioners. In addition, podcasts can encourage online communication through social media networks (Wrather, 2016) and enable practitioners to feel more personally connected to professionals and experts in their field (Lee \& Chan, 2007). Thus, "communities of practice" can emerge around educational podcast usage, whereby knowledge sharing occurs between and among practitioners who share and discuss podcast content in the workplace and online (Wrather, 2016).

Although there is literature to support the use of educational podcasts, there appears to be a dearth of scholarly work focused on the use and impact of educational podcasts within the field of kinesiology (McNamara \& Haegele, 2020). Understanding how the podcasting phenomena is impacting specific fields and professions is essential, as this medium has the ability to be used as a knowledge translation tool to reach a larger audience and increases the likelihood of the use of pertinent information within a particular field (Rader et al., 2014). For instance, because of lower levels of physical activity and increased obesity, new and effective modes of communication must be identified that can effectively inform sedentary populations about the merits of physical activity and health, as well as how to properly conduct physical activities and lead a healthy lifestyle. Turner-McGrievy and Tate (2011) explained that mobile technologies, including podcasts, may be an especially "useful conduit for delivery of a weight-loss program" (p. 2) due to the tremendous growth in the use of these technologies. Podcasting has the ability to be used not only within educational settings but also as a knowledge translation tool to influence various populations and disciplines within the field of kinesiology. Indeed, the potential for this tool, which has been deemed to be unique in its authenticity, accessibility, and ability to instill a community is largely still unknown (McNamara \& Haegele, 2020; Kay, 2012; Lee \& Chan, 2007; McNamara \& Drew, 2019; Meserko, 2015; Wrather, 2016), especially within specific fields (e.g., kinesiology, health promotion) and disciplines (e.g., physical education, sport science).

Although research examining the application and impact on areas within kinesiology appears to be limited, preliminary research suggests it may be an effective learning tool. For example, Mcnamara et al. (2020) recently examined the effectiveness of three educational podcasts that were developed using adult learning theory and cognitive theory of multimedia principles (Mayer, 2011). The podcasts were developed to inform 29 special education administrators how to properly supervise and monitor adapted PE services for students with disabilities. Results showed a small, but significant, increase in test scores from pre- to posttest. Furthermore, the majority of the participants had positive perceptions toward the usefulness of the podcasts. This investigation suggests that educational podcasts may have some merit as a tool to disseminate information about the field of kinesiology to other key stakeholders. However, because of the limited amount of peerreviewed literature dedicated to the use of educational podcasts within research and professions within the field of kinesiology (McNamara \& Haegele, 2020), additional research and exploration on this topic are needed.

\section{Purpose}

Although the quantity of research focused on educational podcasts is increasing (e.g., Kay, 2012; Prakash, Muthuraman, \& Anand, 2017; Wilson, Petticrew, \& Booth, 2009), little is known about the impact and use of educational podcasts to disseminate information with regard to kinesiology content (McNamara \& Haegele, 2020). This is problematic, as O'Bannon, Lubke, Beard, and Britt (2011) explained that because educational podcasting research "is in its infancy, there is a need for additional research in different content areas and for longer periods of time" (p. 1891) in order to determine the long-term impact of this new tool. Scoping reviews are a particularly useful research methodology when the literature on a certain topic is broad and heterogeneous, as they allow for the wide ranging exploration of a specific topic (Arksey \& O'Malley, 2005; Tricco et al., 2016). Scoping reviews enable researchers to chart the foundational concepts underpinning a broad research topic "and the main sources and types of evidence available" (Tricco et al., 2016, p. 2). Although similar to a systematic review, scoping reviews make it possible to present a comprehensive overview of the literature pertaining to a topic, irrespective of study quality, and are especially useful when examining scholarly areas that are emerging to clarify concepts and identify gaps within the literature (Peters et al., 2015; Tricco et al., 2016). Recently, scoping reviews have become more commonly used in response to a growing demand for summaries of the breadth of research around a particular topic (Goertzen, et al., 2015); this method of research has also been increasingly prevalent within the field of kinesiology (e.g., Aloraini et al., 2020; Goertzen, et al., 2015; Killian, Kinder, $\&$ Woods, 2019). The purpose of the current study was to conduct a scoping review of the literature in order to capture a comprehensive view of the peer-reviewed research, as well as the peer-reviewed practitioner writings on the use of educational podcasts in the field of kinesiology. In addition, this paper sought to identify any existing gaps in the literature. The following research questions guided this scoping review:

(a) What research has been conducted that has examined the use of educational podcasts to disseminate information with regard to kinesiology content?

(b) What scholarly practitioner articles exist related to the use of educational podcasts in the field of kinesiology?

(c) What gaps exist within the literature related to educational podcasting and the field of kinesiology?

\section{Method}

The scoping review methodological framework proposed by Arksey and O'Malley (2005) and the Preferred Reporting Items for Systematic Reviews extension for Scoping Reviews Checklist (Tricco et al., 2018) guided this investigation. A recent scoping review focused on online and blended learning within the field of PE (Killian et al., 2019) was used to guide the development of the research questions and study selection process for the present scoping review. For this scoping review, podcasts were defined as a medium that delivers digital content through audio and visual mediums that can be distributed via the Internet to a variety of media devices such as smartphones and laptops (Hew, 2008). Although the definition of the field of kinesiology is contested (Reeve, 2007; Schary \& Cardinal, 2016), for the purposes of this scoping review, kinesiology was defined as the study of human movement and physical activity (Reeve, 2007). This includes the production, dissemination, and application of kinesiology-related knowledge, within relevant professional contexts (e.g., physical educators, sport psychologists, physical therapists; Reeve, 2007). A broad definition of kinesiology was able to capture a comprehensive overview of research that has disseminated information related to the field of kinesiology. 


\section{Eligibility Criteria}

The following inclusion criteria were used to identify relevant articles: (a) published between January 2003 and December 2019; (b) published in English language journals; (c) located in periodical publications (i.e., research articles and practitioner articles); (d) podcasts were included and discussed within the paper; (e) focused on a field within kinesiology (e.g., physical therapy, PE, exercise science). The year 2003 was starting point for the review as Berry (2015) noted that this year marked the conception of podcasts. For this study, peer-reviewed practitioner writing refers to peer-reviewed articles that are absent of methodology and raw data, and are given to support practitioners in a variety of ways including advising, establishing best practices, and noting trends.

\section{Search Strategy}

A university librarian conducted a search of the literature using the following databases: CINAHL, Communication \& Mass Media Complete, Education Full Text, ERIC, PubMed, and SPORTDiscus. Search terms for all databases included a sophisticated search strategy that utilized Boolean Operators (i.e., and, or, not) and truncation (i.e., the use of the asterisk to retrieve various word endings). This search was conducted using different platforms (e.g., EBSCO, ProQuest) as each has their own unique searching capabilities and scope. To ensure a comprehensive search, the search term syntax used for this study included terms from either a podcast or a kinesiology category (podcast* OR vodcast* OR webcast* OR "streaming technology" OR "video casting" OR videocasting AND Kinesiology OR "physical education and training" OR "sport science" OR "exercise science" OR "movement science" OR "physical activity” OR "adapted physical activity” OR "sport psychology" or "sport management" OR "medical education" OR "community health"). This search term syntax was designed to cast the widest net possible, which led to the overidentification of citations.

\section{Study Selection Process}

According to Preferred Reporting Items for Systematic Reviews extension for Scoping Reviews Checklist, scoping reviews should utilize a data extraction process, which is commonly referred to as the data charting process (Killian et al., 2019; Peters et al., 2015; Tricco et al., 2018). First, each abstract, title, and authors' names of all articles collected within the database searches were inserted into a Google Sheets data charting file. Second, all duplicate articles were removed. Next, the articles were evaluated using a two-step process. The investigators first conducted independent title and abstract reviews of the identified articles to ensure they met inclusion criteria and were relevant to the review. After the title and abstract search, the investigators carefully reviewed each article to confirm that they met the full inclusion criteria. For both steps, at least two investigators independently evaluated all articles. In instances of disagreement, a third investigator who served as a tiebreaker reassessed articles. The interrater agreement between the investigators was calculated at $95 \%$.

The data charting tool used in this study identified key characteristics of the included articles. Data were extracted from each article and entered into the appropriate form by the authors. Data extracted from research studies were organized according to the following items: (a) research design, (b) podcast features, (c) study research questions and/or purpose, (d) data collection methods, (e) participant characteristics, and (f) key results. These items were selected to determine the extent to which specific characteristics were reported in the included articles. In addition, a basic numerical analysis with similar data categories as described previously was performed with the empirical research to illustrate trends and gaps within the literature (Arksey \& O'Malley, 2005; Killian et al., 2019). Three of the investigators reviewed each of the included articles to confirm that the information for each item was accurate.

\section{Results}

A total of 802 articles were initially identified through the database searches and reference list searches. There were 464 duplicate articles that were excluded. Next, the investigators screened article titles and abstracts, which resulted in the exclusion of an additional 286 articles for failure to meet eligibility criteria. Next, the authors conducted full reviews of the remaining 52 articles, and an additional 38 articles were excluded. Once the review process was completed, 14 articles met the full eligibility criteria. Figure 1 provides an overview of the article identification process. Table 1 presents an overview of the research studies $(n=11)$, as well as a numerical analysis of the characteristics of the empirical articles identified are provided in Table 2.

\section{Research Articles}

\section{Research Design}

Of the 14 articles that met the inclusion criteria, 11 were datadriven research articles. Within these articles, a variety of research designs were implemented. Seven studies implemented an experimental/quasi-experimental design, with four of these employing experimental randomized control designs. Of the remaining four articles, one used a qualitative design, two used a mixed-methods design, and one used a Delphi method. All but one of the research studies focused on the impact of podcasts on either learning, motivation, or a physiological change (e.g., heart rate, weight loss). This outlier study used a Delphi method with a range of health professionals (e.g., occupational therapists, emergency medicine specialists) aimed to identify quality markers for blogs and podcasts within health-related fields (Lin et al., 2015).

\section{Design and Implementation of Podcasts}

The implementation of the podcasts in the identified research studies varied widely. Nine studies used investigator-created podcasts, and one study (Turner-McGrievy et al., 2009) compared investigator-created podcasts with a series of open-access podcasts. Three of these studies incorporated "vodcasts," which are podcasts that utilize visuals (Abt \& Barry, 2007; Hurst, 2016; McNeill \& Fry, 2012). McNeill and Fry (2012) used video clips from course activities and used them as the foundation of the vodcasts. In addition, they compared the perceived usefulness of the vodcasts with audio podcasts.

Four studies identified the social cognitive theory (SCT; Bandura, 1991) as a theoretical framework that was used to develop the podcasts employed in these studies. All studies that used the SCT examined participant weight loss (Hales et al., 2017; TurnerMcGrievy et al., 2009; Turner-McGrievy \& Tate, 2011; TurnerMcGrievy et al., 2017). Two studies (Hurst, 2016; McNeill \& Fry, 2012) related their findings to learning theories. The remaining 

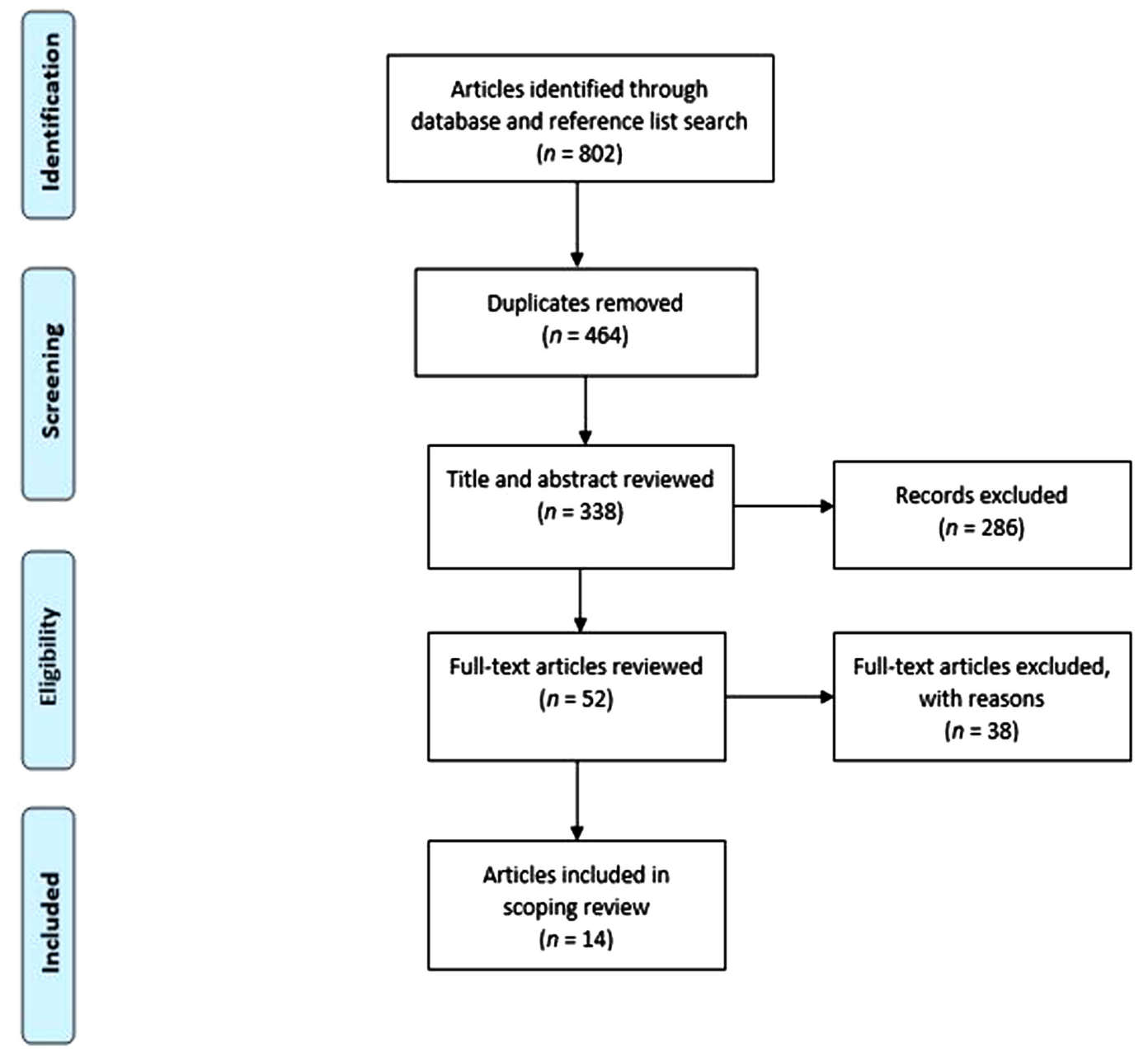

Figure 1 - PRISMA flow diagram of scoping review search.

studies had no reference to specific theories in relation to podcast development or their findings (Abt \& Barry, 2007; Lin et al., 2015; McNeill, Mukherjee, \& Singh, 2010; Rockhill, Pastore, \& Johnston, 2019; Stork, Karageorghis, \& Ginis, 2019). Five studies addressed the design and development of the podcast(s) incorporated within the studies (McNeill et al., 2010; McNeill \& Fry, 2012; Turner-McGrievy et al., 2009; Turner-McGrievy \& Tate, 2011).

Two of the studies utilized podcasts as a supplementary component of their studies (Hales et al., 2017; Turner-McGrievy et al., 2017). For example, Hales and colleagues (2017) examined the predictors of weight loss and points earned on a weight loss application in obese and overweight adults. Although informational podcasts were delivered to participants twice a week throughout the duration of the study, this was given to both the control group and experimental group, and thus was not the main intervention. The eight remaining quantitative, qualitative, and mixed-methods studies used podcasts as a primary tool within their investigations (Abt \& Barry, 2007; Hurst, 2016; McNeill et al., 2010; McNeill \& Fry, 2012; Rockhill et al., 2019; Stork et al., 2019; Turner-McGrievy et al., 2009; Turner-McGrievy \& Tate, 2011). However, within Stork and colleagues' (2019) study, podcasts, as well as no audio, were used as a control in order to examine the effectiveness of motivational music on psychological and physiological responses to an exercise protocol.

\section{Participants}

Sample sizes ranged from 16 to 96 participants. Overall, the included studies had 552 total participants. Five studies examined undergraduate or postgraduate students in kinesiology-related courses. This included students enrolled in PE courses (McNeill et al., 2010; McNeill \& Fry, 2012), exercise physiology courses (Abt \& Barry, 2007), physiotherapy courses (Hurst, 2016), and sports management courses (Rockhill et al., 2019). Five studies focused on overweight or obese participants (Hales et al., 2017; Stork et al., 2019; Turner-McGrievy et al., 2009; Turner-McGrievy \& Tate, 2011; Turner-McGrievy et al., 2017), and one study surveyed health profession educators, which included occupational therapists (Lin et al., 2015).

\section{Data Collection and Outcomes}

Outcome measures for the included studies focused largely on weight loss and the listeners' perceptions toward the usefulness of the podcasts. Of the five studies that focused on weight loss, each collected information related to body mass index, weight, amount and level of physical activity, and food consumption. Other data collected included personality characteristics (Hales et al., 2017; Turner-McGrievy et al., 2009), eating behaviors (TurnerMcGrievy \& Tate, 2011), points earned on a weight loss application (Hales et al., 2017), self-efficacy (Turner-McGrievy \& Tate, 


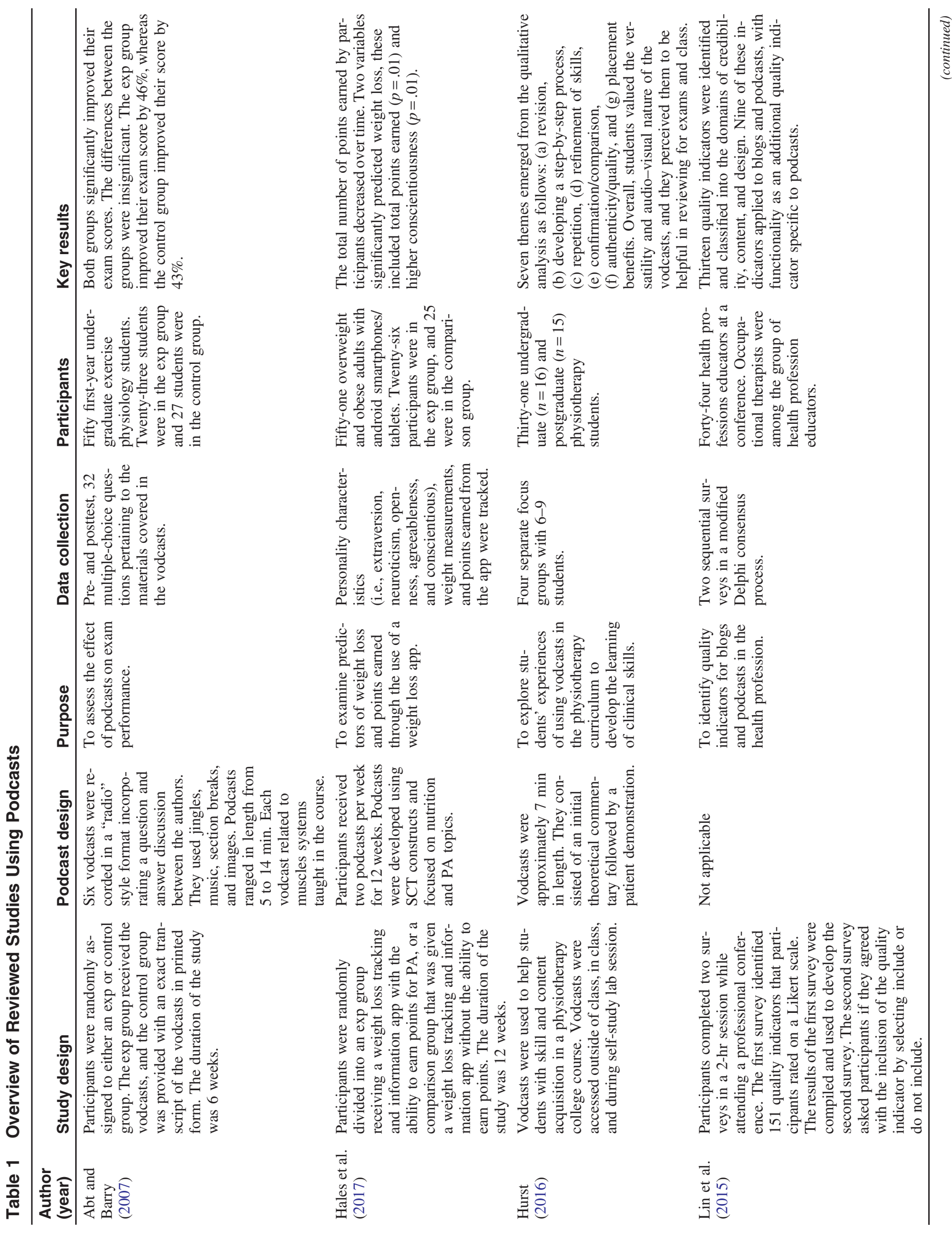




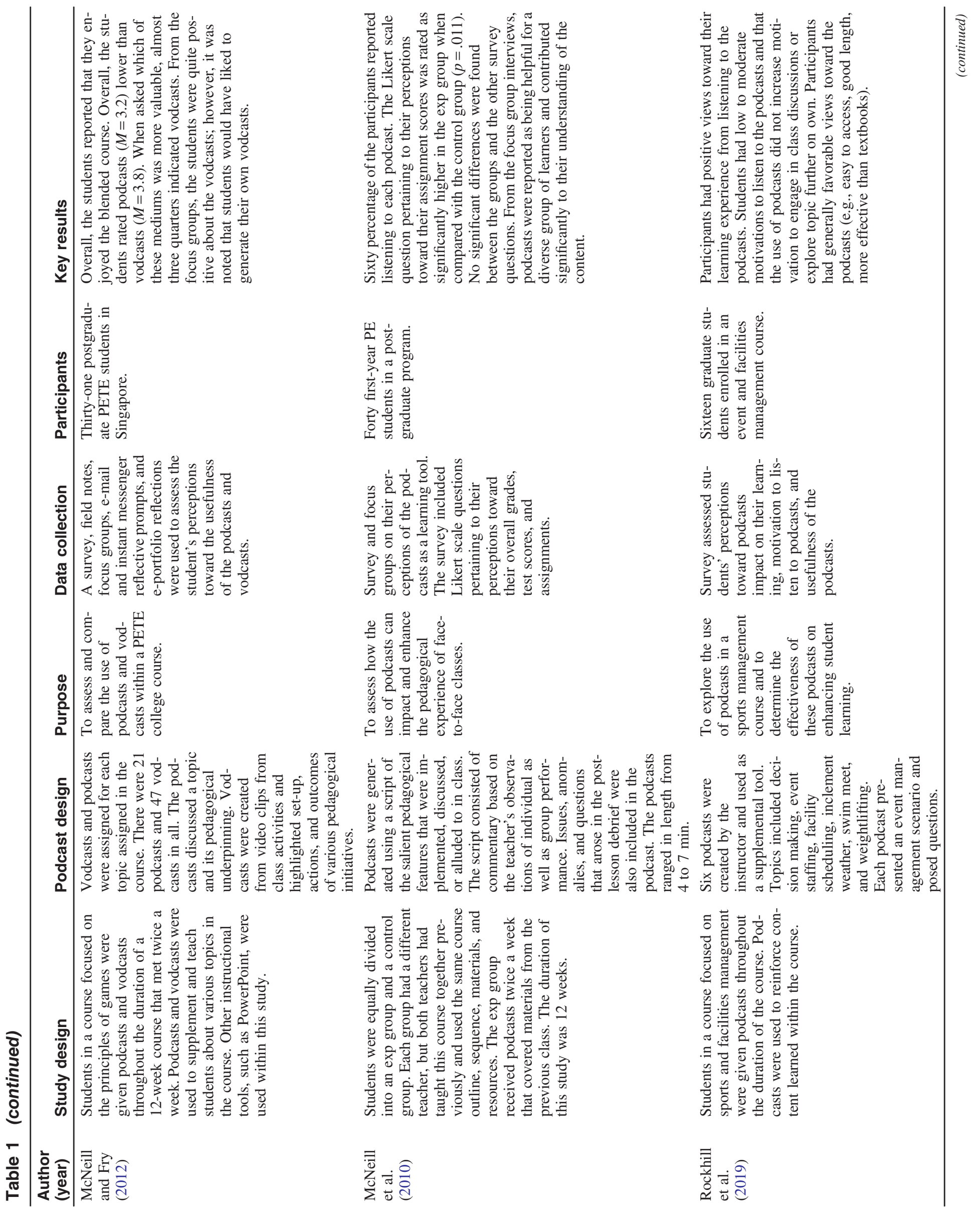




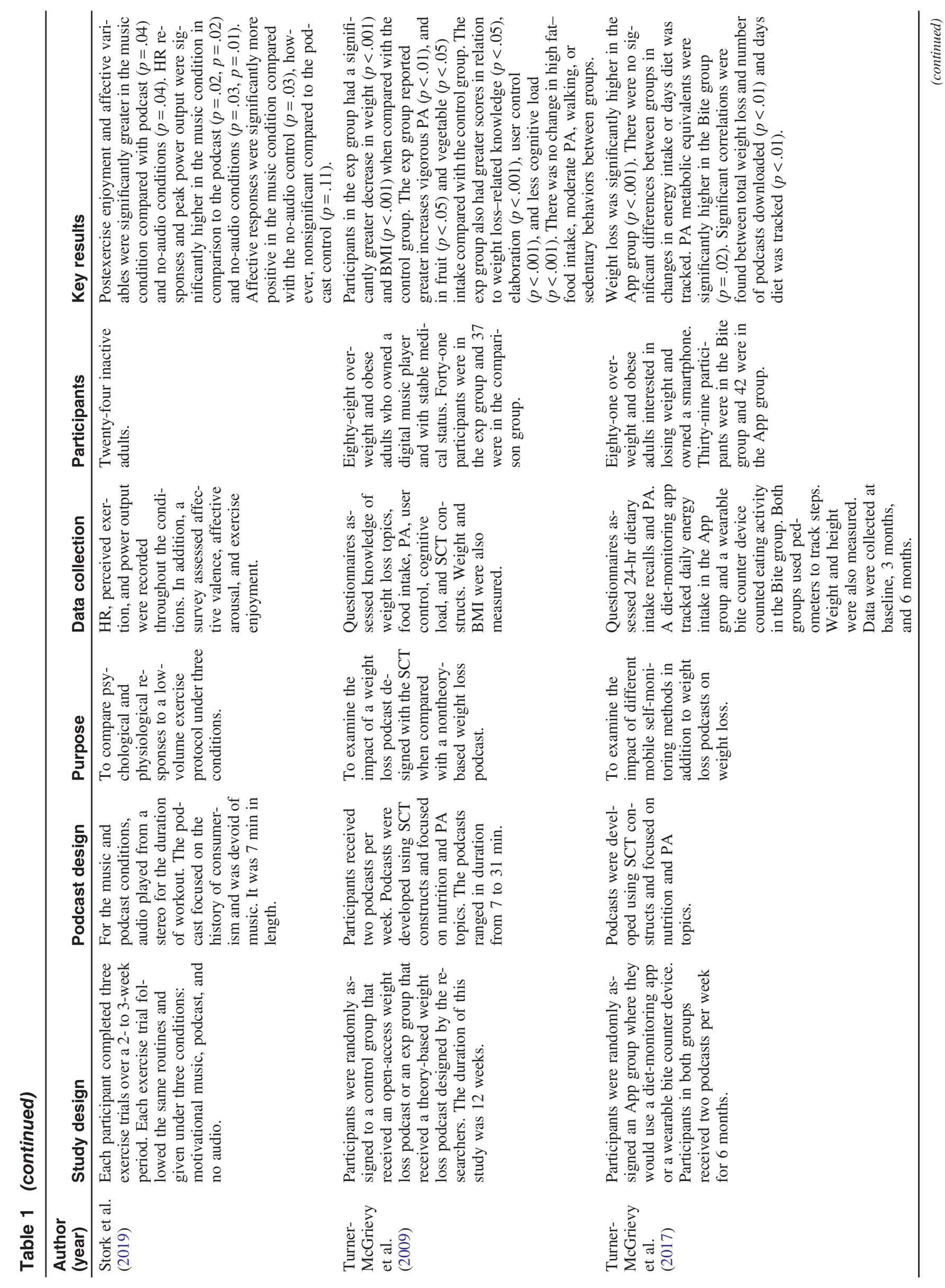




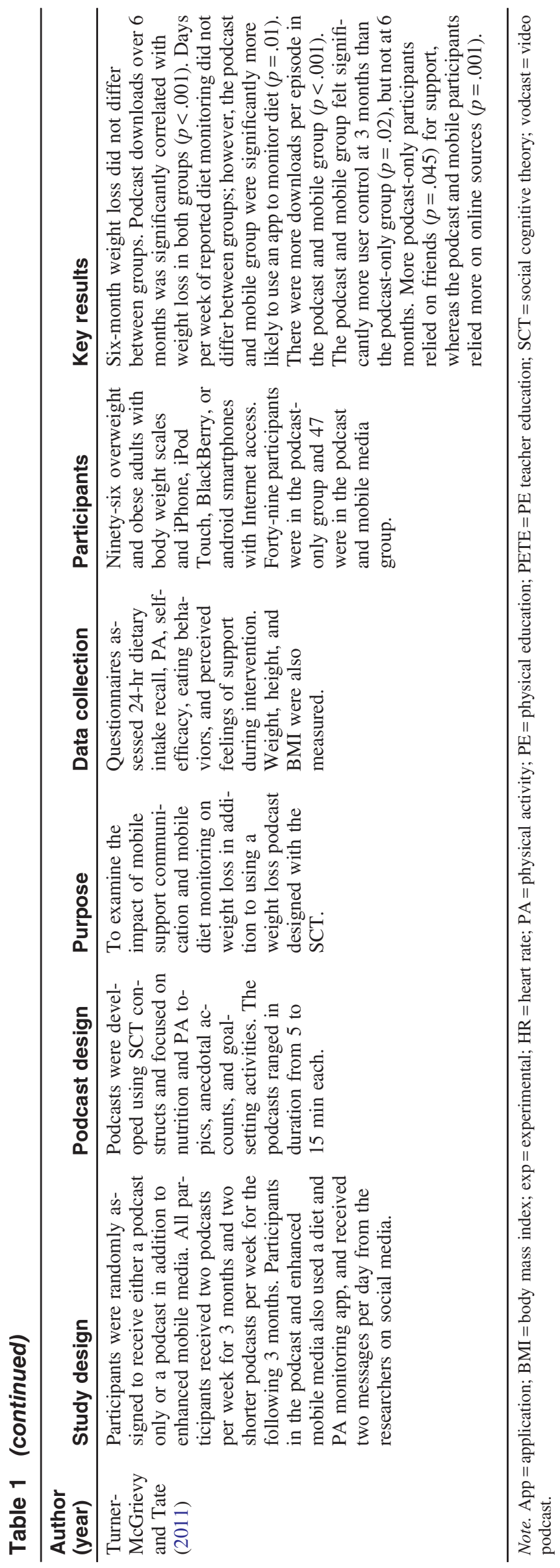

KR Vol. 10, No. 1, 2021 
Table 2 Numerical Analysis of the Research Articles

\begin{tabular}{|c|c|c|c|c|c|c|c|c|c|c|c|}
\hline & 1 & 2 & 3 & 4 & 5 & 6 & 7 & 8 & 9 & 10 & 11 \\
\hline \multicolumn{12}{|l|}{ Research design } \\
\hline Experimental/quasi-experimental & $x$ & $x$ & & & & & $x$ & $x$ & $x$ & $x$ & $x$ \\
\hline Qualitative & & & $x$ & & & & & & & & \\
\hline Mixed method & & & & & $x$ & $x$ & & & & & \\
\hline Delphi method & & & & $x$ & & & & & & & \\
\hline \multicolumn{12}{|l|}{ Area(s) examined } \\
\hline Learning & $x$ & & $x$ & & $x$ & $x$ & $x$ & & $\times$ & & \\
\hline Motivation & & & & & $x$ & & $x$ & $x$ & & & \\
\hline Physiological change & & $\times$ & & & & & & $x$ & $\times$ & $x$ & $x$ \\
\hline \multicolumn{12}{|l|}{ Participants } \\
\hline Obese/sedentary & & $\times$ & & & & & & $x$ & $\times$ & $\times$ & $x$ \\
\hline College students & $x$ & & $x$ & & $x$ & $x$ & $x$ & & & & \\
\hline Health profession educators & & & & $\times$ & & & & & & & \\
\hline \multicolumn{12}{|l|}{ Podcast type(s) } \\
\hline Investigator created & $x$ & $\times$ & $x$ & $x$ & $x$ & $x$ & $x$ & & & $\times$ & $x$ \\
\hline Open access & & & & & & & & $x$ & $\times$ & & \\
\hline Vodcasts & $x$ & & $\times$ & & $x$ & & & & & & \\
\hline \multicolumn{12}{|l|}{ Theory used } \\
\hline SCT & & $x$ & & & & & & & $\times$ & $x$ & $x$ \\
\hline CTML & & & $x$ & & & & & & & & \\
\hline CTL & & & & & $x$ & & & & & & \\
\hline None & $x$ & & & $x$ & & $x$ & $x$ & $x$ & & & \\
\hline \multicolumn{12}{|l|}{ Primary data collected } \\
\hline Knowledge test & $x$ & & & & & & & & $x$ & & \\
\hline Survey & & $x$ & & $x$ & $\times$ & $x$ & $x$ & $x$ & $\times$ & $x$ & \\
\hline Focus groups & & & $x$ & & $x$ & $x$ & & & & & \\
\hline Weight loss points & & $x$ & & & & & & & $x$ & $x$ & $x$ \\
\hline BMI & & & & & & & & & $x$ & $\times$ & $x$ \\
\hline Heart rate & & & & & & & & $x$ & & & \\
\hline
\end{tabular}

Note. 1 = Abt \& Barry (2007); 2 = Hales et al. (2017); 3 = Hurst (2016); 4 = Lin et al. (2015); 5 = McNeill \& Fry (2012); 6= McNeill et al. (2010); $7=$ Rockhill et al. (2019); $8=$ Stork et al. (2019); 9 = Turner-McGrievy et al. (2009); $10=$ Turner-McGrievy \& Tate (2011); $11=$ Turner-McGrievy et al. (2017); CTL = constructivist learning theory; $\mathrm{CTML}=$ cognitive theory of multimedia learning; $\mathrm{SCT}=$ social cognitive theory; $\mathrm{BMI}=$ body mass index.

2011), and knowledge tests via the podcasts (Turner-McGrievy et al., 2009; Turner-McGrievy \& Tate, 2011). Stork and colleagues (2019) collected other forms of physiological data, which included heart rate and power output during an exercise protocol.

Within the weight loss studies, it appears that these were extensions of one another. Turner-McGrievy and colleagues (2009) first examined the impact of podcasts on knowledge and weight loss between a group that received podcasts grounded within the SCT (Bandura, 1991) and another group that received open-access podcasts. Participants who were provided the theory-driven podcasts significantly outperformed the open-access podcast group on several measures, such as weight loss, amount of physical activity, and knowledge scores. Next, Turner-McGrievy and Tate (2011) examined the difference between participants who received a podcast intervention and a group that received a podcast and a mobile device intervention. Both of these podcasts were developed using the SCT. There was no significant difference in weight loss. The two more recent studies have incorporated the podcasts as a supplementary or supporting component of the intervention (Hales et al., 2017; Turner-McGrievy et al., 2017). Furthermore, within these more recent studies, there is no explicit podcast development section; rather there is just a reference to the podcast development protocol from the 2009 and 2011 studies (Turner-McGrievy et al., 2009; Turner-McGrievy \& Tate, 2011). All four weight loss studies resulted in significant weight loss due to the use of an intervention that utilized podcasts. However, it should be reemphasized that two of these studies used podcasts as a supplementary tool (Hales et al., 2017; Turner-McGrievy et al., 2017). Thus, when reviewing the results of the weight loss studies, it may be generalized that the use of podcasting of weight loss information, in accompaniment of other online interventions (e.g., weight loss applications) enhances obese and overweight individuals' ability to lose weight.

Five research studies examined how college kinesiology students are impacted by and perceive podcasts as an educational tool (Abt \& Barry, 2007; Hurst, 2016; McNeill et al., 2010; McNeill \& Fry, 2012; Rockhill et al., 2019). Of the studies that occurred within college settings, only the study conducted by Abt and Barry (2007) used podcasts as a typical intervention within their study, with the other studies simply embedding the podcasts within their classes and examining their impact on students. More specifically, Abt and Barry (2007) used a pretest/posttest design to measure knowledge gained from the use of podcasts. In this study, 
the experimental group received podcasts while the intervention group received transcripts of the podcasts. This study found that both groups improved significantly in their test scores, but there was no significant difference between groups. From the focus groups, participants expressed that the podcasts were flexible and helpful for a diverse group of learners, and they contributed significantly to their understanding of the content. For example, one participant stated, "I liked being able to constantly look back, rewind it, and replay it whenever I wanted to. This is good for any kind of learner" (Hurst, 2016, p. 208). This was reiterated for vodcasts by one participant explaining "they are awesome" (McNeill \& Fry, 2012). McNeill and colleagues (2010) also employed a survey to measure the students' perceptions toward the usefulness of the podcasts in relation to their grades. When asked if the podcasts were helpful, 15\% responded with "ok," 50\% with "very helpful," and 40\% as "exceptionally helpful." The most popular responses from open-ended questions about the usefulness of the podcasts consisted of the aspect of consolidation, reiteration, and reinforcement of the content. In addition, there were no identified significant differences between the two groups in their perceptions toward their overall grades or test scores; however, the podcast group did have significantly higher perceptions toward their assignment grades. Overall, these results suggest that kinesiology students perceive educational podcasts as a flexible, accessible, and beneficial tool to their learning; however, there is little quantitative evidence to directly show any substantial impact of podcasts on their learning.

Lin and colleagues (2015) implemented a modified Delphi method with 44 health profession educators, which included occupational therapists, to come to a consensus on quality indicators for both blogs and podcasts. Thirteen quality indicators that classified into the domains of credibility $(n=8)$, content $(n=4)$, and design $(n=1)$ met the inclusion threshold. Nine of these indicators applied to both blogs and podcasts, with an additional three indicators specific to blogs and one specific to podcasts. Examples of the indicators that were specific to both blogs and podcasts include "Do the authorities (e.g., author, editor, and publisher) that created the resource list their conflicts of interest?" (Credibility) and "Is the content of this educational resource of good quality?" (Content). The design indicator was only applicable to podcasts. This indicator reinforced the importance of functionality of podcasts for all learners. The podcast design indicator was "Does the resource employ technologies that are universally available to allow learners with standard equipment and software access?" The authors concluded that "by identifying the quality indicators most important to health professions educators, this modified Delphi study provides 13 quality indicators that may help develop standards, guide development, and improve identification of high-quality medical education blogs and podcasts" (p. 549).

\section{Practitioner Articles}

Three practitioner articles qualified for inclusion in the scoping review (McNamara \& Brooks, 2016; Mears, 2009; Shumack \& Reilly, 2011). Each of these articles was quite similar in several aspects. Each focused on using podcasts within a PE setting and were published within PE practitioner journals $(1=$ Strategies: $A$ Journal for Physical and Sport Educators, 2 = Journal of Physical Education, Recreation and Dance). In addition, each article described the beneficial use of podcasts within a PE setting, as well as each provided a systematic outline on how to create podcasts and listed the equipment needed to properly record podcasts. Shumack and Reilly (2011) focused solely on vodcasts, whereas the other two articles focused on both audio and video podcasts (McNamara \& Brooks, 2016; Mears, 2009). In addition, McNamara and Brooks (2016) listed available open-access podcasts that focus on PE topics.

\section{Discussion}

The purpose of the current study was to conduct a scoping review of the literature in order to capture a comprehensive view of the peer-reviewed research, as well as the peer-reviewed practitioner writings on the use of educational podcasts in the field of kinesiology. Fourteen articles were identified and evaluated. Based on the findings of this scoping review, it appears that most of the literature reports positive overall findings concerning the use of educational podcasts within the field of kinesiology. Another major finding from this scoping review is that there is a general lack of peer-reviewed literature that has examined educational podcasts within the field of kinesiology. This finding displays a divergence between the literature and the field. Other fields, especially within the medical field, have had a much greater presence of educational podcasting literature (e.g., Prakash et al., 2017; Wilson et al., 2009). In fact, three articles identified within this scoping review were published within medical journals (Lin et al., 2015; Turner-McGrievy et al., 2009; Turner-McGrievy \& Tate, 2011). Several studies in the medical education literature have utilized podcasts for a variety of purposes. For example, Prakash and colleagues (2017) explained that podcasts are an efficient teaching method, even in comparison to other modes of learning, such as traditional face-to-face teaching or text-based learning. Researchers and practitioners within the field of kinesiology should consider engaging with discipline-specific podcasts, producing their own podcasts, or collaborating with existing podcast producers to further disseminate their research findings and best practices. Some journals now host their own podcast, which include discussions and summaries of content published in the journal, as well as discussion on current and contemporary topics. Journals extending their media platforms include British Journal of Sports Medicine and the Clinical Journal of Sport Medicine. PD organizations and educational institutions may also find it worthwhile to consider developing their own podcasts to deliver esoteric knowledge to their communities and help to provide kinesiology professions with ongoing content. This will provide an opportunity for researchers and practitioners to disseminate knowledge in a way that is engaging for both professional and college audiences, as well as meet the need for flexibility and availability in a busy and drastically changing world.

Although this scoping review provides a comprehensive overview of the evidence and research trends, the heterogeneity of the studies and limited literature on this topic make it difficult to form any substantial conclusions. However, the simple recognition of this gap in the literature may be important to the field of kinesiology, as it may allow for more concerted efforts to examine and document the value of this medium. In an attempt to have more concentrated endeavors to examine the impact of educational podcasting within the field of kinesiology, certain aspects from this scoping review should be recognized. For one, all of the literature was focused either on participants receiving a physical activity intervention or on college students. However, research on practitioners within the field of kinesiology (e.g., physical educators, coaches, physical therapists) was 
entirely missing from this review. This is concerning, as educational podcasts have been identified to be a valuable tool for continuing PD (Healy et al., 2020; Kennedy et al., 2017). In addition, for practitioners within the field of kinesiology, podcasts may be an especially advantageous learning tool, as this group has often cited financial and time constraints to attending formal PD (York et al., 2014).

However, it should be noted that one recently published study examined the use of educational podcasts with in-service physical educators (Healy et al., 2020). This study was excluded from the current scoping review as it was not indexed within the databases during the timeframe from which this scoping review search occurred. For this study, Healy and colleagues (2020) conducted a quasi-experimental study to determine the effectiveness of online training for physical educators. The asynchronous (i.e., self-paced) online PD was developed using adult learning theory and cognitive theory of multimedia principles (Mayer, 2011). The online PD consisted of video podcasts, sought to teach physical educators how to properly implement peer tutoring in order to better include students with disabilities within a PE setting. Results showed the experimental group that used educational podcasts had significantly higher posttest scores on how to properly implement peer tutoring compared with the waitlist control group. Furthermore, the majority of the participants in the experimental group had positive perceptions toward the online setting for receiving education. This investigation suggests that educational podcasts can be an effective learning tool for practitioners within the field of kinesiology. Research such as this is essential, as it suggests that educational podcasts can be an effective learning tool for practitioners within the field of kinesiology. This may provide insight on using educational podcasts as a tool in the future to provide both college students and practitioners within the field of kinesiology the opportunity to easily access quality PD through mediums outside of traditional face-toface PD, which has been cited to have significant barriers to regularly attending (York et al., 2014).

More studies should explicitly discuss the development and design process of the podcasts utilized within their studies, as many of the studies within this review failed to explicitly state this process and the content within the podcasts. For example, several of the articles reviewed had no mention of whether the podcasts used were designed by the investigators or were pre-existing openaccess podcasts. The absence of this information prohibits future researchers from using similar methodologies in developing highquality podcasts. The lack of overall depth of research protocols prevalent throughout many of the studies identified within the present review. These findings are consistent with Kay (2012) who argued that it is crucial to confront these "significant methodological concerns in order to establish the reliability and validity of results, compare and contrast results from different studies, and address some of the more difficult questions such as under what conditions and with whom" (p. 826) podcasts are most useful. This absence of methodological reliability and validity is especially problematic as it can lead to a lack of confidence in the results.

Aside from the weight loss-focused studies (e.g., TurnerMcGrievy et al., 2009), the studies identified within the scoping review did not appear to adhere to any specific theoretical frameworks to guide the design of the podcasts. Other educational podcast researchers in related areas have also raised this concern (Hew, 2008; Mayer, 2011; Mcnamara et al., 2020). Another concern is that the majority of educational podcast studies are action-based research studies that mostly lack any discussion of theoretical frameworks (Drew, 2017; Hew, 2008; McNamara \& Drew, 2019; Mcnamara et al., 2020). This also aligns with a great deal of the research identified within this review (e.g., McNeill et al., 2010; McNeill \& Fry, 2012). In addition, other than the studies that focused primarily on weight loss, most of the identified studies examined participants' enjoyment and engagement with regard to podcasting rather than focusing on understanding how learning is occurring. McNamara and Drew (2019) recently stated this is problematic, as "although it is important to measure participants' enjoyment and motivations while listening to podcasts for learning purposes, it is crucial that researchers also begin to examine the cognitive learning outcomes associated with listening to educational podcasts" (p. 5). Hence, research explicitly focused on cognitive learning outcomes is warranted in order to gain a comprehensive understanding of the impact of podcasts used for PD or within college courses.

It is noteworthy that the same scholar (i.e., Turner-McGrievy) was a key contributor in four of the 11 data-driven articles identified. This author, along with other authors, appears to be continuing a line of inquiry that exemplifies the significance impact educational podcasts can have to inform and motivate overweight and obese people. This series of studies has led to refined podcast development and implementation sections that would enable practitioners and researchers alike to better replicate these studies for their own purposes. In addition, many of these studies were paired with a mobile application used to track weight loss. Due to the large increases in obesity across the United States, it is essential that an array of effective modes is identified that can effectively inform sedentary populations about the merits of physical activity and health, as well as how to properly conduct physical activities and lead a healthy lifestyle. It appears that Turner-McGrievy and colleagues have begun a strong research line that is able to create a more comprehensive understanding of how the podcasting phenomena, along with mobile devices, might be employed as a knowledge translation tool to affect overweight and obese populations seeking to lose weight. Scholars should strive to conduct studies that progress off one another to discern how the podcasting phenomena can be employed as a knowledge translation tool to influence various populations and disciplines within the field of kinesiology.

\section{Limitations}

The limitations to this scoping review should be acknowledged. First, scoping reviews are limited in that their "focus is to provide breadth rather than depth of information in a particular topic. As such, the conduct of a meta-analysis is generally not conducted in a scoping review" (Tricco et al., 2016, p. 9). Thus, it is difficult to make any widespread generalizations from this review. Second, like most scoping and systematic reviews, this investigation was limited by the inclusion criteria. For example, this review only included studies disseminated in English, due to the large amount of studies initially screened and the investigators' language limitations. Third, the definition of the field of kinesiology used to help guide this investigation should also be noted as a limitation of this study. As noted previously, simply defining the field of kinesiology is controversial, as this is multidisciplinary field that often conducts interdisciplinary research (Reeves, 2007; Schary \& Cardinal, 2016). Therefore, because of the broad scope and multidisciplinary nature of kinesiology, it is difficult to conduct a full review of the relevant articles within each field that falls within kinesiology. 


\section{Conclusions}

The low cost, ease of production, and rapid distribution associated with educational podcasts has made it an increasingly prevalent instructional tool (Kay, 2012; McNamara et al., 2020; Wilson et al., 2009). However, research within the area of educational podcasting in the field of kinesiology appears to be in its infancy. Although this scoping review may be an important starting point in developing this area of literature, it is critical that future researchers report all of the information critical to their research design, instrument development, and findings. For example, future researchers should examine the use of various theoretical frameworks used within the development of specific types of podcasts (e.g., short-duration podcasts, narrative podcasts; McNamara et al., 2020). Future researchers should also explicitly examine the impact and perceived usefulness of educational podcasts with different populations within the field of kinesiology (e.g., in-service practitioners, undergraduate kinesiology students). Even simple descriptive research would be beneficial, as it would allow for an understanding of the extent to which these groups are using this medium to fulfill their PD and learning needs.

\section{References}

Abt, G., \& Barry, T. (2007). The quantitative effect of students using podcasts in a first year undergraduate exercise physiology module. Bioscience Education, 10(1), 1-9. doi:10.3108/beej.10.8

Aloraini, S.M., Gelley, G., Glazebrook, C., Sibley, K.M., Singer, J., \& Passmore, S. (2020). Motor behavior concepts in the study of balance: A scoping review. Journal of Motor Behavior, 52(1), 97-121. PubMed ID: 30888258 doi:10.1080/00222895.2019.1582472

Arksey, H., \& O’Malley, L. (2005). Scoping studies: Towards a methodological framework. International Journal of Social Research Methodology, 8(1), 19-32. doi:10.1080/1364557032000119616

Bandura, A. (1991). Social cognitive theory of self-regulation. Organizational Behavior and Human Decision Processes, 50(2), 248-287. doi:10.1016/0749-5978(91)90022-1

Beddoes, Z.E., Prusak, K.A., \& Barney, D. (2019). Taking the helm: Physical educators managing change through professional learning communities. Quest, 71(4), 479-496. doi:10.1080/00336297.2018. 1556169

Berry, R. (2015). A golden age of podcasting? Evaluating Serial in the context of podcast histories. Journal of Radio \& Audio Media, 22(2), 170-178. doi:10.1080/19376529.2015.1083363

Blackett, A.D., Evans, A., \& Piggott, D. (2017). Why 'the best way of learning to coach the game is playing the game': Conceptualising 'fasttracked' high-performance coaching pathways. Sport, Education and Society, 22(6), 744-758. doi:10.1080/13573322.2015.1075494

Drew, C. (2017). Educational podcasts: A genre analysis. E-Learning and Digital Media, 14(4), 201-211. doi:10.1177/2042753017736177

Fronek, P., Boddy, J., Chenoweth, L., \& Clark, J. (2016). A report on the use of open access podcasting in the promotion of social work. Australian Social Work, 69(1), 105-114. doi:10.1080/0312407x. 2014.991338

Goertzen, L., Halas, G., Rothney, J., Schultz, A.S., Wener, P., Enns, J.E., \& Katz, A. (2015). Mapping a decade of physical activity interventions for primary prevention: A protocol for a scoping review of reviews. JMIR Research Protocols, 4(3), 1-7. doi:10.2196/resprot.4240

Hales, S., Turner-McGrievy, G.M., Wilcox, S., Davis, R.E., Fahim, A., Huhns, M., \& Valafar, H. (2017). Trading pounds for points: Engagement and weight loss in a mobile health intervention. Digital Health, 3, 1-9. doi:10.1177/2055207617702252
Healy, S., Block, M., \& Kelly, L. (2020). The impact of online professional development on physical educators' knowledge and implementation of peer tutoring. International Journal of Disability, Development and Education, 67(4), 424-436. doi:10.1080/1034912x.2019.1599099

Hew, K.F. (2008). Use of audio podcast in K-12 and higher education: A review of research topics and methodologies. Educational Technology Research and Development, 57(3), 333-357. doi:10.1007/ s11423-008-9108-3

Hurst, K.M. (2016). Using video podcasting to enhance the learning of clinical skills: A qualitative study of physiotherapy students' experiences. Nurse Education Today, 45, 206-211. PubMed ID: 27552715 doi:10.1016/j.nedt.2016.08.011

Kay, R.H. (2012). Exploring the use of video podcasts in education: A comprehensive review of the literature. Computers in Human Behavior, 28(3), 820-831. doi:10.1016/j.chb.2012.01.011

Kennedy, M.J., Rodgers, W.J., Romig, J.E., Mathews, H.M., \& Peeples, K.N. (2017). Introducing the content acquisition podcast professional development process: Supporting vocabulary instruction for inclusive middle school science teachers. Teacher Education and Special Education, 41(2), 140-157. doi:10.1177/0888406417745655

Killian, C.M., Kinder, C.J., \& Woods, A.M. (2019). Online and blended instruction in $\mathrm{K}-12$ physical education: A scoping review. Kinesiology Review, 8(2), 110-129. doi:10.1123/kr.2019-0003

Lave, J., Wenger, E., \& Wenger, E. (1991). Situated learning: Legitimate peripheral participation. Cambridge, United Kingdom: Cambridge University Press.

Lee, M.J.W., \& Chan, A. (2007). Reducing the effects of isolation and promoting inclusivity for distance learners through podcasting. Turkish Online Journal of Distance Education, 8(1), 85-105.

Lin, M., Thoma, B., Trueger, N.S., Ankel, F., Sherbino, J., \& Chan, T. (2015). Quality indicators for blogs and podcasts used in medical education: Modified Delphi consensus recommendations by an international cohort of health professions educators. Postgraduate Medical Journal, 91(1080), 546-550. PubMed ID: 26275428 doi:10.1136/ postgradmedj-2014-133230

Mayer, R.E. (2011). Applying the science of learning. Boston, MA: Pearson.

Mcnamara, S., \& Brooks, C. (2016). Promoting physical education through podcasts. Journal of Physical Education, Recreation \& Dance, 87(6), 50-52. doi:10.1080/07303084.2016.1192928

Mcnamara, S., Dillon, S., Becker, K., Healy, S., \& Trujillo-Jenks, L. (2020). The impact of podcasts on special education administrators' understanding of adapted physical education services. International Journal of Disability, Development and Education. Advance online publication. doi:10.1080/1034912X.2020.1731437.

Mcnamara, S., \& Drew, C. (2019). Concept analysis of the theories used to develop educational podcasts. Educational Media International, 56(4), 300-312. doi:10.1080/09523987.2019.1681107

McNamara, S.W.T., \& Haegele, J.A. (2020). Undergraduate students' experiences with educational podcasts to learn about inclusive and integrated physical education. European Physical Education Review. Advance online publication. doi:10.1177/1356336X20932598

McNeill, M., Mukherjee, S., \& Singh, G. (2010). Podcasting in physical education teacher education. ICHPER-SD Journal of Research, 5(1), $16-19$.

McNeill, M.C., \& Fry, J.M. (2012). The value of ICT from a learning gameplaying perspective. ICHPER-SD Journal of Research, 7(2), 45-51.

Mears, D. (2009). Podcasts and Wiki's: Delivering content information to students using technology. Article\# 4 in a 6-Part Series. Strategies, 23(1), 29-34.

Meserko, V.M. (2015). The pursuit of authenticity on Marc Maron's WTF podcast. Continuum, 29(6), 796-810. doi:10.1080/10304312.2015. 1073682 
O’Bannon, B.W., Lubke, J.K., Beard, J.L., \& Britt, V.G. (2011). Using podcasts to replace lecture: Effects on student achievement. Computers \& Education, 57(3), 1885-1892. doi:10.1016/j.compedu.2011.04.001

Peters, M.D., Godfrey, C.M., Khalil, H., McInerney, P., Parker, D., \& Soares, C.B. (2015). Guidance for conducting systematic scoping reviews. International Journal of Evidence-Based Healthcare, 13(3), 141-146. PubMed ID: 26134548 doi:10.1097/xeb.000000000000 0050

Prakash, S.S., Muthuraman, N., \& Anand, R. (2017). Short-duration podcasts as a supplementary learning tool: Perceptions of medical students and impact on assessment performance. BMC Medical Education, 17(1), 1-14.doi:10.1186/s12909-017-1001-5

Rader, T., Pardo, J.P., Stacey, D., Ghogomu, E., Maxwell, L.J., Welch, V.A., ... Tugwell, P. (2014). Update of strategies to translate evidence from Cochrane musculoskeletal group systematic reviews for use by various audiences. The Journal of Rheumatology, 41(2), 206-215. PubMed ID: 24293571 doi:10.3899/jrheum.121307

Reeve, T.G. (2007). Kinesiology: Defining the academic core of our discipline: Introduction. Quest, 59(1), 1-4.doi:10.1080/00336297. 2007.10483531

Rockhill, C., Pastore, D., \& Johnston, D. (2019). The effectiveness of podcasts in sport management education. Journal of Hospitality, Leisure, Sport \& Tourism Education, 25, 1-7. doi:10.1016/j.jhlste. 2019.100211

Sato, T., \& Haegele, J.A. (2018). Physical educators' engagement in online adapted physical education graduate professional development. Professional Development in Education, 44(2), 272-286.doi:10.1080/ 19415257.2017.1288651

Schary, D.P., \& Cardinal, B.J. (2016). Starting to uncover the mystery of interdisciplinary research in kinesiology. Physical Educator, 73(2), 173-184.doi:10.18666/tpe-2016-v73-i2-6184

Shumack, K.A., \& Reilly, E. (2011). Video podcasting in physical education. Journal of Physical Education, Recreation \& Dance, 82(1), 39-43.

Stork, M.J., Karageorghis, C.I., \& Ginis, K.A.M. (2019). Let's Go! Psychological, psychophysical, and physiological effects of music during sprint interval exercise. Psychology of Sport and Exercise, 45, 1-10.
Tricco, A.C., Lillie, E., Zarin, W., O’Brien, K., Colquhoun, H., Kastner, M., ... Straus, S.E. (2016). A scoping review on the conduct and reporting of scoping reviews. BMC Medical Research Methodology, 16(1), 1-10. doi:10.1186/s12874-016-0116-4

Tricco, A.C., Lillie, E., Zarin, W., O’Brien, K.K., Colquhoun, H., Levac, D., ... Straus, S.E. (2018). PRISMA extension for scoping reviews (PRISMA-ScR): Checklist and explanation. Annals of Internal Medicine, 169(7), 467-473. PubMed ID: 30178033 doi:10.7326/m180850

Turner-McGrievy, G., \& Tate, D. (2011). Tweets, apps, and pods: Results of the 6-month Mobile Pounds off Digitally (Mobile POD) randomized weight-loss intervention among adults. Journal of Medical Internet Research, 13(4), 1-14. doi:10.2196/jmir.1841

Turner-McGrievy, G.M., Campbell, M.K., Tate, D.F., Truesdale, K.P., Bowling, J.M., \& Crosby, L. (2009). Pounds off digitally study: A randomized podcasting weight-loss intervention. American Journal of Preventive Medicine, 37(4), 263-269. doi:10.1016/j.amepre.2009. 06.010

Turner-McGrievy, G.M., Wilcox, S., Boutté, A., Hutto, B.E., Singletary, C., Muth, E.R., \& Hoover, A.W. (2017). The dietary intervention to enhance tracking with mobile devices (DIET Mobile) study: A 6-month randomized weight loss trial. Obesity, 25(8), 1336-1342. doi:10.1002/oby.21889

Valian, E., \& Emami, H. (2013). Online education: Definitions and advantages in adult education. Life Science Journal, 10(6), 614-622.

Wilson, P., Petticrew, M., \& Booth, A. (2009). After the gold rush? A systematic and critical review of general medical podcasts. Journal of the Royal Society of Medicine, 102, 69-74. PubMed ID: 19208871 doi:10.1258/jrsm.2008.080245

Wrather, K. (2016). Making 'maximum fun' for fans: Examining podcast listener participation online. Radio Journal: International Studies in Broadcast \& Audio Media, 14(1), 43-63. doi:10.1386/rjao.14.1. 43_1

York, R., Gastin, P., \& Dawson, A. (2014). What about us? We have careers too! The career experiences of Australian sport scientists. International Journal of Sports Science \& Coaching, 9(6), 14371456.doi:10.1260/1747-9541.9.6.1437 